\title{
ECOLOGIA DE MOSQUITOS (DIPTERA, CULICIDAE) EM CRIADOUROS NATURAIS E ARTIFICIAIS DE ÁREA RURAL DO NORTE DO PARANÁ, BRASIL. II. COLETAS COM ISCA HUMANA ${ }^{1}$
}

\author{
José Lopes ${ }^{2}$ \\ Ana L. Lozovei ${ }^{3}$
}

\begin{abstract}
ECOlogy of mosquitols (Diptera, CulicidaE) IN NATURAL AND ARTIFICIAL BREEDING SITES OF TIIE RURAL AREA IN NORTII PARANÁ, BRAZIL. II. CAPTURE OF HUMAN BAIT. With then purpose of knowing the species of mosquitos that colonize an anthropogenic area in the North of Paraná, Brazil. 1496 specimens were captured by the humam bait method, accountig 23 species among them the following were predominant: Anopheles strodei Root, 1926; An. evansae Brethes, 1926; An. galvaoi Causey, Deane \& Deane, 1943; An albitarsis Lynch Arribalzaga, 1878; Coquillettidea juxtamansonia Chagas, 1907; Co. venezuelensis Theobaldi, 1912; Culex (Melanoconion) sp. e Aedes fluviatilis (Lutz, 1904) and showed shong anthopophilia and a high degree of adaptation to humam environment. On the cantray, the species that showed litth adaptation to such ambients or to the geoclimatic conditions of the region were: Anopheles triannulatus (Neiva \& Pinto, 1922); An. parvus (Chagas, 1907); An. lutzii Cruzi, 1901, Culex amazonensis (Lutz, 1905); Cx. chidesteri; Psorophora confinnis (Linch Arribalzaga, 1891); Ps. discrucians (Walker, 1856); Ps. cingulata (Fabricius, 1805) e Aedes scapularis. Although Anopheles argyritarsis Robineau-Desvoidy, 1827; Culex coronator Dyar \& Knab, 1906; Cx. mollis Dyar \& Knab, 1906 and $C x$. quinquefasciatus Say, 1823, were captured in little numbers, it is knowntht they an mosquito of human surroundings. These mosquitos showed a plak of feeding activity directly related to sunset bat did not have a bimodal behaviour. Anophelinae were in general more active in spring while Culex Linnaeus, 1758; Coquillettidia Dyar, 1905; Aedes Meigen, 1818; Mansonia Blanchard, 1901 e Psorophora Robineau-Desvoidy, 1827 were more active in summer.

KEY WORDS. Culicidae, ecology, human bait, behaviour feeding, population fluctuation
\end{abstract}

Transformações ambientais artificiais repentinas direcionam populações animais, ou parte delas com potencial genético para tanto, à novas adaptações em ambientes degradados, podendo aproximá-las das populações humanas. Os mosquitos, assim como todos os outros animais silvestres na região, foram e continuam sendo envolvidos neste contexto de transformações.

1) Parte da Tese de Doutorado do autor sênior.

2) Departamento de Biologia Animal e Vegetal, Universidade Estadual de Londrina. Caixa Postal 6001, 86051-970 Londrina, Paraná, Brasil.

3) Departamento de Patologia, Universidade Federal do Paraná. Centro Politécnico, 81530-900 Curitiba, Paraná, Brasil. 
Tendo como premissa o desenvolvimento e as transformações ambientais do norte do Paraná, foi executada pesquisa visando conhecer as espécies que procriam nas águas de um ribeirão, e dentre elas aquelas que são aptas a se reproduzirem em recipientes alocados na mata ciliar e até mesmo nos recipientes do peridomicílio rural daquela área. Simultaneamente foram coletados mosquitos adultos através de isca humana, procurando identificar outras espécies que não tenham sido coletas na forma de larvas, inferir sobre o grau de antropofilia, verificar a existência de flutuação populacional, evidências de adaptações ao ambiente antrópico e possível domiciliação e determinar o horário do pico de atividade hematofágica.

Estas informações poderão contribuir para a ecologia das espécies coletadas e indicar possíveis avanços adaptativos no sentido da domiciliação.

\section{MATERIAL E MÉTODOS}

O trabalho foi realizado numa área rural no Município de Cambé, localizado na região Norte do Estado do Paraná, Brasil. A descrição da área foi abordada por LOPES \& LOZOVEI (1995).

A estação de coleta foi montada no Sítio do Engenho, na margem esquerda do Ribeirão São Domingos, onde um represamento formava uma lagoa que era a segunda de uma série de cinco, formadas em consequência de represamentos. Neste ponto não havia mata ciliar e a lagoa tinha suas margens internas recobertas por vegetação aquática. Corresponde a estação de coleta número 3 descrita por LOPES \& LOZOVEI (1995).

As coletas de Culicidae adultos usando isca humana, foram realizadas mensalmente no período compreendido entre junho de 1988 a junho de 1989, com duração de 25 horas para cada coleta, perfazendo um total de 325 horas.

Dois coletores sentados e tendo as pernas e pés expostos, localizavam, com a iluminação de uma lanterna, os mosquitos que pousavam para picar. Os coletores-isca foram revezados a cada 2 horas, procurando-se repetir as mesmas pessoas nas diferentes coletas e sempre no mesmo horário. Mosquitos que pousavam em partes do corpo, que não as pernas e pés, também foram aproveitados, acontecendo inclusive o fato de um coletor capturar no corpo do outro, quando o acesso era impraticável por parte deste.

Os Culicidae foram capturados com um tubo de ensaio devidamente etiquetado. Ao final de cada período de 15 minutos de coleta, os tubos de captura eram trocados e identificados para permitir análise temporal da atividade de picada.

Vários exemplares puderam ser aprisionados num mesmo tubo, cada um ficando separado do outro por chumaços de algodão.

Após as 25 horas ininterruptas de coletas, os mosquitos foram transportados ao laboratório, sacrificados com vapor de éter etílico e montados em pontas para posterior identificação.

Após a identificação, um lote de exemplares foi encaminhado ao Dr. Oswaldo Paulo Forattini, da Faculdade de Saúde Pública, Universidade de São Paulo, para confirmação específica. 
O material entomológico identificado está depositado na coleção entomológica da Universidade Estadual de Londrina.

\section{RESULTADOS}

Foram coletadas 1496 fêmeas de Culicidae pertencentes a seis gêneros e 23 diferentes espécies (Tab. I). Por esta tabela observa-se que nos meses de junho e julho de 1988 , não foi coletado nenhum culicídeo, no entanto o mês de setembro foi o de maior incidência.

Tabela I. Total mensal para cada espécie, coletada com isca humana, às margens do Ribeirão São Domingos, Cambé, Paraná, de junho de 1988 à junho de 1989. Legenda: (Ans) Anopheles strodei, (Ang) An. galvaoi, (Ane) An. evansae, (Aal) An. albitarsis, (Ant) An. triannulatus, (Anp) An. parvus, (Anl) An. lutzii, (Ano) An. oswaldoi, (Ana) An. argyritarsis, (Cqj) Coquillettidia juxtamansonia, (Cqv) Co. venezuelensis, (Cxc) Culex coronator, (Cxq) Cx. quinquefasciatus, (Cml) Cx. (Melanoconion) sp., (Cxa) Cx. amazonensis, (Cch) Cx. chidesteri, (Cxm) Cx. mollis, (Mat) Mansonia titillans, (Psd) Psorophora discrucians, (Psc) Ps. cingulata, (Pco) Ps. confinnis. (Aef) Aedes fluviatilis. (Aes) Ae. scapularis.

\begin{tabular}{|c|c|c|c|c|c|c|c|c|c|c|c|c|c|c|c|c|c|c|c|c|c|c|c|c|}
\hline \multirow{2}{*}{ Data } & \multicolumn{23}{|c|}{ Espécies } & \multirow{2}{*}{ - Tota } \\
\hline & Ans & Ang & Ane & Aal & Ant & Anp & Anl & Ano & Ana & Cqj & Cqv & $\mathrm{Cxc}$ & $\mathrm{Cxq}$ & $\mathrm{Cml}$ & Cxa & Cch & $C x m$ & $\mathrm{n}$ Mat & Psd & Psc & Pco & Aef & Aes & \\
\hline Jun/88 & 0 & 0 & 0 & 0 & 0 & 0 & 0 & 0 & 0 & 0 & 0 & 0 & 0 & 0 & 0 & 0 & 0 & 0 & 0 & 0 & 0 & 0 & 0 & 0 \\
\hline Jul/88 & 0 & 0 & 0 & 0 & 0 & 0 & 0 & 0 & 0 & 0 & 0 & 0 & 0 & 0 & 0 & 0 & 0 & 0 & 0 & 0 & 0 & 0 & 0 & 0 \\
\hline Ago/88 & 312 & 19 & 0 & 2 & 0 & 0 & 0 & 0 & 0 & 0 & 0 & 0 & 0 & 0 & 0 & 0 & 0 & 0 & 0 & 0 & 0 & 0 & 0 & 33 \\
\hline Set/88 & 319 & 149 & 87 & 14 & 4 & 4 & 2 & 0 & 0 & 3 & 4 & 2 & 1 & 1 & 1 & 2 & 0 & 1 & 0 & 0 & 0 & 0 & 0 & 594 \\
\hline Out/88 & 62 & 22 & 27 & 4 & 0 & 0 & 0 & 0 & 0 & 7 & 2 & 0 & 5 & 1 & 0 & 0 & 0 & 1 & 0 & 0 & 0 & 2 & 1 & 134 \\
\hline Nov/88 & B 46 & 10 & 28 & 3 & 1 & 0 & 0 & 2 & 0 & 8 & 0 & 0 & 1 & 0 & 0 & 0 & 1 & 1 & 0 & 0 & 0 & 1 & 0 & 102 \\
\hline Dez/88 & 375 & 8 & 57 & 1 & 8 & 1 & 2 & 4 & 0 & 64 & 12 & 3 & 4 & 19 & 1 & 0 & 1 & 7 & 0 & 0 & 0 & 4 & 0 & 271 \\
\hline Jan/89 & 12 & 2 & 17 & 0 & 2 & 1 & 0 & 1 & 1 & 8 & 3 & 6 & 1 & 20 & 0 & 1 & 6 & 1 & 5 & 0 & 0 & 11 & 0 & 98 \\
\hline Fev/89 & 2 & 1 & 1 & 2 & 1 & 1 & 0 & 0 & 2 & 15 & 1 & 3 & 0 & 10 & 3 & 0 & 0 & 3 & 0 & 0 & 0 & 14 & 0 & 59 \\
\hline Mar/89 & 17 & 4 & 12 & 3 & 5 & 0 & 0 & 1 & 1 & 31 & 2 & 2 & 0 & 25 & 8 & 0 & 4 & 1 & 0 & 0 & 0 & 2 & 0 & 118 \\
\hline Abr/89 & 6 & 0 & 11 & 1 & 1 & 0 & 0 & 0 & 0 & 18 & 1 & 2 & 0 & 9 & 1 & 0 & 1 & 0 & 0 & 1 & 0 & 2 & 0 & 54 \\
\hline Mai/89 & 6 & 2 & 1 & 0 & 0 & 1 & 0 & 0 & 0 & 0 & 0 & 0 & 0 & 0 & 0 & 0 & 0 & 0 & 0 & 0 & 1 & 1 & 0 & 12 \\
\hline Jun/89 & 7 & 8 & 2 & 0 & 0 & 1 & 0 & 0 & 0 & 0 & 1 & 0 & 0 & 2 & 0 & 0 & 0 & 0 & 0 & 0 & 0 & 0 & 0 & 21 \\
\hline Total & 564 & 25 & 243 & 30 & 22 & 9 & 4 & 8 & 4 & 154 & 26 & 18 & 12 & 87 & 14 & 3 & 13 & 15 & 5 & 1 & 1 & 37 & 1 & 1496 \\
\hline
\end{tabular}

Todos os espécimens de Culex (Melanoconion) identificados aproximaramse a vexans. Em trabalho concomitante realizado por LOPES \& LOZOVEI (1995) com capturas de larvas no Ribeirão São Domingos, encontraram quatro espécies de Melanoconion Theobald, 1903. Assim, para evitar erro na identificação, a espécie passa a ser referida como $C x$. (Melanoconion) sp.

O gênero Anopheles Meigen, 1818 foi predominante contribuindo com $74,1 \%$ do total de espécimes coletados, e dentro deste gênero, Anopheles strodei Root, 1926, foi a espécie com maior freqüência contribuindo com $37,7 \%$ de todos os mosquitos coletados. Nos meses de janeiro a abril de 1989 houve diminuição no número de exemplares do gênero Anopheles coletados.

Anopheles strodei foi mais abundante no meses de setembro a dezembro de 1988 e em maio e junho de 1989. Nesse último mês esteve com densidade populacional semelhante ao de Anopheles galvaoi Causey, Deane \& Deane, 1943 que também foi a mais expressivo no mês de agosto. No mês de janeiro a espécie 
mais coletada foi de $C x$. (Melanoconion) sp. e nos meses de fevereiro a abril foi de Coquillettidia juxtamansonia (Chagas, 1907), embora essa freqüência tenha sido igualada por Aedes fluviatilis (Lutz, 1904) no mês de fevereiro.

Analisando-se a tabela I observa-se que An. strodei, An. galvaoi, An. evansae (Brethes, 1926), An. albitarsis Lynch Arribalzaga, 1878 e An. parvus (Chagas, 1907) foram mais abundantes no mês de setembro com suas populações concentradas de setembro a dezembro. As duas espécies de Coquillettidia Dyar, 1905 tiveram maiores densidades populacionais no mês de dezembro, mantendo-se elevada até abril. A mesma distribuição observa-se em Culex coronator Dyar \& Knab, 1906 e $C x$. (Melanoconion) sp., embora o maior número de indivíduos tenha sido coletado em janeiro para a primeira espécie e em março para a segunda. Aedes fluviatilis foi coletada de outubro de 1988 a maio de 1989 , apresentando pico populacional em janeiro e fevereiro.

As espécies de Anopheles em geral, com exceção à An. triannulatus (Neiva \& Pinto, 1922) mais abundante no verão, atingiram maiores densidades populacionais na primavera. As espécies de Culex Linnaeus, 1758, Coquillettidia e Aedes Meigen, 1818 foram mais abundantes no verão, com exceção ao subgênero Aedinus do gênero Culex, que apresenta maior freqüência de captura no outono (Tab. II).

Tabela II. Distribuiçăo estacional para os gêneros e espécies de Culicidae coletados com isca humana nas margens do Ribeirão São Domingos, Cambé, Paraná, de junho de 1988 à junho de 1989. Legenda: (An.) Anopheles, (Cx) Culex, (Co) Coquillettidia, (Ma) Mansonia, (Ps) Psorophora, (Ae) Aedes, (Ans) An. strodei, (Ang) An. galvaoi, (Ane) An. evansae, (Aal) An. albitarsis, (Ant) An. triannulatus, (Anp) An. parvus, (Cqj) Co. juxtamansonia, (Cqv) Co. venezuelenis. (C(C)) Culex (Culex). (C(M)) Culex (Melanoconion). (C(A)) Culex (Aedinus).

\begin{tabular}{|c|c|c|c|c|c|c|c|c|c|c|c|c|c|c|c|c|c|c|}
\hline \multirow{2}{*}{ Estações } & \multirow{2}{*}{ Culicidae } & \multicolumn{6}{|c|}{ Gêneros } & \multicolumn{11}{|c|}{ Espécies } \\
\hline & & An & $C x$ & Co & $\mathrm{Ma}$ & PS & $\mathrm{Ae}$ & Ans & Ang & Ane & Aal & Ant & Anp & Cqj & Cqv & $C(C)$ & $\mathrm{C}(\mathrm{M})$ & $C(A)$ \\
\hline Inverno & 56 & 53 & 2 & 1 & 0 & 0 & 0 & 21 & 27 & 2 & 2 & 0 & 1 & 0 & 1 & 0 & 2 & 0 \\
\hline Primavera & 824 & 778 & 17 & 24 & 2 & 0 & 3 & 427 & 181 & 142 & 19 & 5 & 4 & 18 & 6 & 14 & 2 & 1 \\
\hline Verăo & 422 & 192 & 82 & 103 & 11 & 5 & 29 & 89 & 11 & 75 & 3 & 11 & 3 & 87 & 16 & 29 & 49 & 4 \\
\hline Outono & 190 & 70 & 60 & 52 & 1 & 2 & 5 & 29 & 6 & 24 & 4 & 6 & 1 & 49 & 3 & 19 & 32 & 9 \\
\hline
\end{tabular}

A tabela III globaliza os resultados das coletas, agrupando-os em intervalos de trinta minutos, não apresentando no entanto, os resultados das coletas no período diurno por não haver sido coletado nenhum culicídeo. A maior ocorrência de mosquitos foi observada entre 18:30 e 21:00 horas.

A figura 1 apresenta o horário dos picos, admitindo um prolongamento de 15 minutos em cada coleta e o número de pernilongos coletados neste período. Os horários dos picos foram sendo retardados até dezembro e, a partir daí, começou a sofrer antecipação. As variações, em geral foram de 15 minutos. Há uma tendência de aumento na quantidade de Culicidae capturados, quanto mais tarde se evidecia o pico de atividade hematofágica, mas estando sempre correlacionado com o crep. Este comportamento está correlacionado com a duração do dia luz. Quanto maior o período de claridade, tanto mais tarde aparece o pico de atividade hematofágica 
dos Culicidae e, devido às maiores temperaturas nestes meses, a população destes mosquitos tende a ser maior.

As espécies de Anopheles foram coletadas praticamente durante toda a noite, mas revelaram nítida predominância no período vespertino. Os Aedes foram capturados nos crepúsculos e nas auroras com predominância no primeiro. Os espécimes do gênero Psorophora Robineau-Desvoidy, 1827 só foram coletados até às 19:45 horas, no entanto as dos gêneros Coquillettidic e Culex tenderam a uma distribuição durante toda a noite, mas com significativa concentração no crepúsculo.

Tabela III. Total de Culicidae, das treze capturas, distribuidos por intervalos de 30 minutos, em coletas com isca humana nas margens do Ribeirão São Domingos, Cambé, Paraná, de junho de 1988 à junho de 1989. Legenda: (Ans) Anopheles strodei, (Ang) An. galvaoi, (Ane) An. evansae, (Aal) An. albitarsis, (Ant) An. triannulatus, (Aef) Aedes fluviatilis, (Cqj) Coquillettidia iuxtamansonia. (Cav) Co. venezuelensis (Cml) Culex (Melanoconion) so.

\begin{tabular}{|c|c|c|c|c|c|c|c|c|c|c|c|}
\hline \multirow{2}{*}{ Horas } & \multicolumn{11}{|c|}{ Espécies } \\
\hline & Ans & Ang & Ane & Aal & Ant & Aef & Cqj & Cqv & $\mathrm{Cml}$ & Outros * & Total \\
\hline $18: 00$ & 14 & 11 & 3 & 0 & 0 & 1 & 0 & 1 & 2 & 3 & 35 \\
\hline $18: 30$ & 78 & 40 & 40 & 5 & 4 & 3 & 26 & 2 & 14 & 14 & 226 \\
\hline $19: 00$ & 102 & 40 & 39 & 4 & 4 & 14 & 26 & 5 & 19 & 13 & 266 \\
\hline $19: 30$ & 103 & 35 & 71 & 3 & 2 & 9 & 34 & 14 & 14 & 30 & 315 \\
\hline $20: 00$ & 64 & 18 & 35 & 3 & 1 & 4 & 28 & 3 & 5 & 6 & 167 \\
\hline $20: 30$ & 52 & 14 & 17 & 1 & 4 & 2 & 10 & 0 & 4 & 9 & 113 \\
\hline $21: 00$ & 33 & 10 & 8 & 2 & 3 & 1 & 6 & 0 & 4 & 3 & 70 \\
\hline $21: 30$ & 30 & 12 & 16 & 2 & 2 & 0 & 1 & 0 & 3 & 5 & 71 \\
\hline $22: 00$ & 22 & 12 & 4 & 2 & 0 & 0 & 0 & 0 & 3 & 0 & 45 \\
\hline $22: 30$ & 10 & 5 & 6 & 1 & 0 & 0 & 1 & 0 & 0 & 3 & 26 \\
\hline $23: 00$ & 5 & 5 & 1 & 1 & 0 & 0 & 0 & 1 & 0 & 1 & 14 \\
\hline $23: 30$ & 5 & 1 & 0 & 1 & 0 & 0 & 0 & 0 & 1 & 1 & 9 \\
\hline $00: 00$ & 9 & 5 & 0 & 0 & 0 & 0 & 1 & 0 & 2 & 4 & 21 \\
\hline $00: 30$ & 5 & 0 & 0 & 0 & 0 & 0 & 3 & 0 & 0 & 0 & 8 \\
\hline $01: 00$ & 3 & 2 & 1 & 0 & 0 & 0 & 0 & 0 & 0 & 4 & 10 \\
\hline $01: 30$ & 2 & 0 & 0 & 1 & 0 & 0 & 1 & 0 & 1 & 1 & 6 \\
\hline $02: 00$ & 5 & 3 & 2 & 0 & 1 & 0 & 0 & 0 & 0 & 2 & 13 \\
\hline $02: 30$ & 5 & 0 & 1 & 1 & 0 & 0 & 1 & 0 & 0 & 1 & 9 \\
\hline $03: 00$ & 9 & 3 & 3 & 0 & 0 & 0 & 1 & 0 & 0 & 1 & 17 \\
\hline 03:30 & 2 & 0 & 0 & 0 & 0 & 0 & 1 & 0 & 1 & 0 & 4 \\
\hline $04: 00$ & 0 & 0 & 0 & 0 & 0 & 0 & 0 & 0 & 0 & 0 & 0 \\
\hline $04: 30$ & 0 & 0 & 0 & 0 & 0 & 0 & 0 & 0 & 0 & 1 & 1 \\
\hline $05: 00$ & 3 & 1 & 2 & 0 & 0 & 0 & 4 & 0 & 0 & 1 & 11 \\
\hline $05: 30$ & 3 & 0 & 1 & 0 & 0 & 1 & 3 & 0 & 3 & 1 & 12 \\
\hline $06: 00$ & 1 & 0 & 2 & 1 & 1 & 2 & 5 & 1 & 9 & 8 & 30 \\
\hline $06: 30$ & 0 & 0 & 0 & 0 & 0 & 0 & 0 & 0 & 1 & 2 & 3 \\
\hline
\end{tabular}

*. Outros inclui as espécies: Anopheles oswaldoi, An. parvus, An. lutzii, An. argyritarsis, Aedes scapulares, Psorophora discrucians, Ps. cingulata, Mansonia titillans, Culex coronator, Cx. amazonensis, Cx. mollis, Cx. quinquefasciatus.

\section{DISCUSSÃO}

Aedes fluviatilis apresentou maior atividade hematofágica no verão, restringindo-se praticamente ao crepúsculo, não mostrando comportamento bimodal definido e pouca antropofilia. Distribuição temporal semelhante foi observada por Neves \& Silva (1976). 


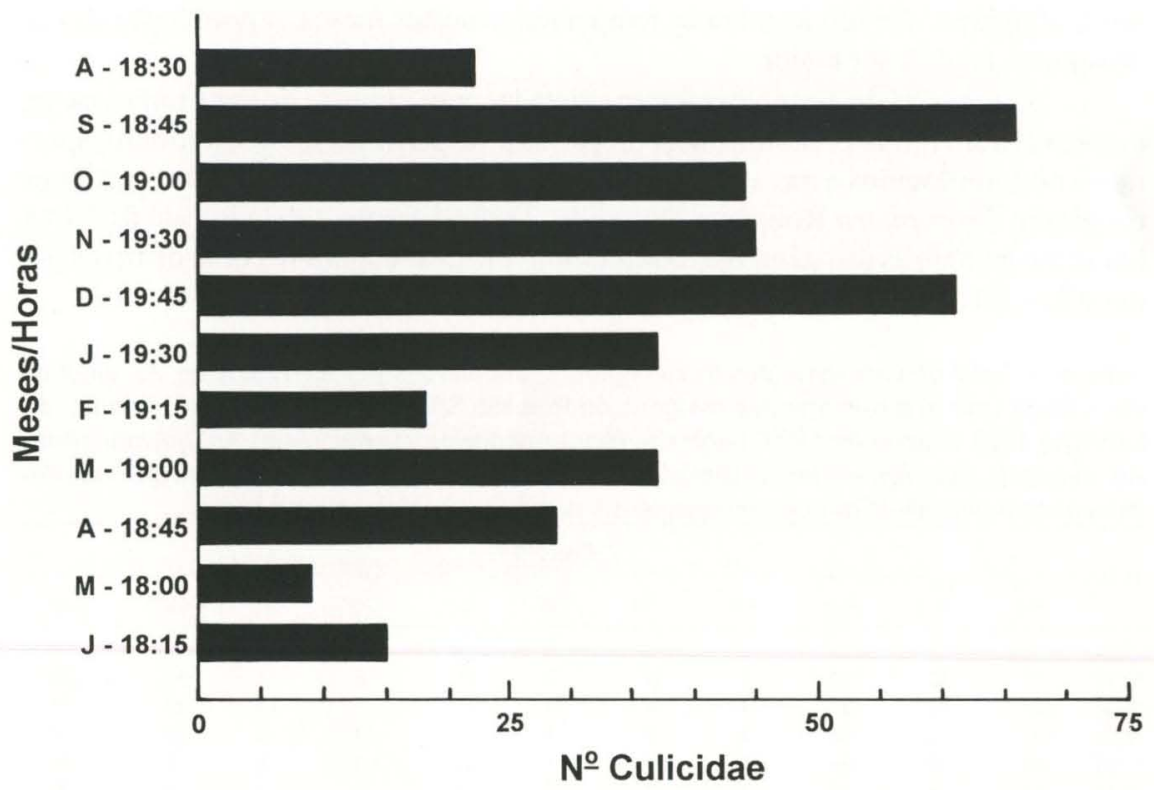

Fig. 1. Número de Culicidae coletados no horário do pico máximo de atividade hematofágica, nas capturas mensais realizadas com isca humana.

Forattini et al. (1968), Neves \& Silva (1976), Consoli \& Williams (1981) demonstraram atividades hematofágicas para Ae. fluviatilis preferencialmente diurna. Neste experimento isso pode não ter ocorrido devido as coletas terem sido realizadas sob incidência solar direta.

Foi coletado apenas um exemplar de Ae. scapularis e esta espécie não foi encontrada por LOPES \& LOZOVEI (1985), nas coletas realizadas no Ribeirão São Domingos, Paraná.

Aedes scapularis costuma ser dominante nas coletas realizadas em ecótopos antropogênicos, principalmente nas regiões litorâneas. Suas altas freqüências no peridomicílio e intradomicílio e a sua forte antropofilia permitiram considerá-la como uma espécie em fase adiantada de domiciliação (FORATTINI et al. 1986b, 1987b, 1989; ForATTINI \& GOMEs 1988). Embora essa espécie seja antropófila e endófila, as exigências fisiológicas para a colocação dos seus ovos distanciam-nas do processo de domiciliação nesta área do norte do Paraná. Isso poderia ser possível em regiões topográficas que favoreçam à postura no tipo de criadouro exigido pela espécie.

Estas coletas, realizadas com isca humana, foram efetivadas à margem da estação 3 de coletas ao longo do leito do Ribeirão São Domingos, onde foi capturado um número elevado de larvas de Aedomyia squamipennis Linch Arribalzaga, 1848 (Lopes \& LOzOVEI 1995). Todavia nenhum adulto foi coletado com isca humana. Esse resultado comprova a falta de antropofilia para essa espécie. 
Anopheles strodei, An. galvaoi, An. evansae e An. albitarsis estiveram praticamente ausentes nas coletas realizadas no inverno e mostraram pico populacional no início da primavera, dando-se a seguir uma estabilização da densidade, mas com acentuada queda no verão. Esses resultados reforçam a hipótese de LOPES \& LOZOVEI (1995) de que essas espécies possam passar à estação fria na fase larval, já que foram capturadas em alta densidade durante o inverno. $\mathrm{O}$ mês de setembro propiciou uma coleta de adultos muito acima da média. Esse fato pode indicar uma forte eclosão de adultos oriúndos das larvas do inverno, justamente no final desta estação e início da primavera.

Esses anofelíneos comportaram-se como espécies tipicamente crepusculares, embora apareçam, em baixa densidade durante toda a noite. Apresentaram comportamento de picada unimodal, com início de atividade estimulada pelo crep.

Os dados registrados na presente pesquisa podem indicar uma forte antropofilia para as quatro espécies de anofelíneos, em contraposição à zoofilia, principalmente pelo fato de que, a menos de $100 \mathrm{~m}$ do local das coletas, aproximadamente 20 bovinos reuniam-se sob algumas árvores para passar a noite.

CORREA \& RAMOS (1941) afirmaram que An. evansae não seria antropófila nem domiciliada, embora pudesse ser encontrada no intradomicílio e capturada com isca humana. Anopheles strodei apresenta-se como não-domiciliada e, embora seja considerada zoófila, pode ser coletada com isca humana e encontrada intradomiciliarmente (RACHOU 1958). CORRÊA (1938) encontrou An. strodei na Fazenda Santa Alice, São Paulo, como domiciliada não sendo entretanto, constante a sua presença nas casas o que poderia ser interpretado como domiciliado temporário.

A colonização em áreas abertas pelas quatro espécies não se caracteriza como novidade, pois a presença delas exercendo atividade hematofágica em áreas de mata residual, ambientes alterados, no peridomicílio e até mesmo no domicílio, é um relato comum na literatura (GALVÃo et al. 1937; BARRETO 1938; RACHOU et al. 1958; ForATtini et al. 1968, 1978a, 1978b, 1981, 1986a, 1987b; NEVES \& Silva 1976).

Anopheles oswaldoi (Piryassu, 1922) mostrou-se pouco adaptada às condições antrópicas ou climáticas desta região, ou então seria apenas menos antropófila. Nas coletas realizadas no leito do ribeirão, também esteve em baixa densidade populacional (LOPES \& LOZOVEI 1995)

Anopheles parvus (Chagas, 1907), An. lutzii Cruz, 1901 e An. triannulatus foram espécies pouco freqüentes indicando fraca adaptabilidade à condição geoclimática desta região e ou as condições de mata ciliar tão reduzida, já que foram coletados por Foratini et al. (1968, 1981, 1987b); Neves \& Silva (1976); GUIMARÃES et al. (1985); GUIMARÃES \& VictóRIO (1986) em áreas abertas e até no peridomicílio. Para as duas primeiras não foram encontrado imaturos nas águas do ribeirão (LOPES \& LOZOVEI 1995).

Anopheles argyritarsis Robineau-Desvoidy, 1827, Culex coronator, Cx. mollis Dyar \& Knab, 1906, Cx. quinquefasciatus Say, 1823 e Psorophora cingulata (Fabricius, 1805) são espécies amplamente conhecidas por colonizarem áreas antrópicas, apresentando características evolutivas para a domiciliação. 
Culex coronator e $C x$. mollis foram capturadas em freqüências relativamente baixas, sendo considerandas preferencialmente zoófilos por FORATTINI et al. (1987a) e GOBALDON et al. (1977) que as coletaram com maior constância engurgitadas com sangue de aves.

Com exceção à $C x$. quinquefasciatus, que esteve com maior densidade populacional na primavera, as outras quatro espécies acima mencionadas, comportam-se como veranículas.

GOMES et al. (1987), obtiveram maior sucesso de captura para Cx. quinquefasciatus nas coletas realizadas no domicílio do que no peridomicílio. Na presente pesquisa, essa espécie pode não ter sido abundante porque as coletas foram realizadas em local aberto e distante das residências.

Das cinco espécies acima mencionadas, apenas Psorophora cingulata não foi capturada nas coletas de larvas realizadas por LoPES \& Lozover (1995). Todavia, as encontradas foram sempre em baixa quantidade

Psorophora cingulata é um culicídeo de hábito alimentar preferencialmente diurno e de ambiente sombreado. Sua presença nas coletas com isca humana foi reduzida provavelmente porque estas não foram realizadas dentro da mata ciliar.

Culex amazonensis (Lutz, 1905) apareceu em número reduzido e esboçou comportamento bimodal da atividade de picar. JORDAN (1961) e LOURENÇO-DEOLIVEIRA (1984) coletaram-na somente em alagados com vegetação. Pode-se aventar a hipótese de antropofilia reduzida ou uma baixa adaptabilidade ao habitat alterado desta região de estudo. As ausências dessa epécie nas coletas de larvas realizadas por LOPES \& LOZOVEI (1995) reforça essa hipótese.

Culex chidesteri foi coletada apenas em duas oportunidades. FORATTINI et al. (1973) assinalaram nível acentuado de competição de $C x$. chidesteri com $C x$. quinquefasciatus no ambiente urbano, caracterizando o potencial de domiciliação. GOMES \& FORATTINI (1990) coletaram-na em baixa quantidade em área rural, entendendo eles que possa existir, para esta espécie, um grau de adaptação a ambientes mais urbanizados.

Psorophora discrucians (Walker, 1856) e Psorophora confinnis (Lynch Arribalzaga, 1891) foram espécies pouco freqüentes. As mesmas foram capturadas através de isca humana, em matas residuais e em ambientes modificados, por NEVES \& Silva (1976), ForATtini et al. (1978a, 1978b) e LOURENÇO-DE-OliveIRA (1984). Assim, podem ser considerados mosquitos aptos a colonizar capoeiras nas margens de ribeirões e córregos. Todavia não foram capturadas larvas dessas espécies no Ribeirão São Domingos (LOPES \& LOZOVEI 1995). Os resultados da presente pesquisa podem não significar tratar-se de espécies pouco comuns nesta área, mas sim que as coletas realizadas durante o dia, fora da mata ciliar, podem ter dificultado o encontro de um número mais elevado desses culicídeos, pois os mesmos apresentam hábito alimentar preferencialmente diurno e no interior de mata.

Coquillettidia venesuelensis (Theobaldi, 1912), Co. juxtamansonia e Mansonia titillans (Walker, 1848) tiveram atividade hematofágica predominantemente no verão, com comportamento crepuscular. Este resultado é semelhante ao observado por LOURENÇO-DE-OliveIRA \& SiLVA (1985). 
Entre as duas espécies de Coquillettidia foi verificado uma separação de 45 minutos entre os picos de atividade alimentar e isso pode representar uma forma de evitar competição. LOPES et al. (1995b) estudando os mosquitos do Campus Universitário de Londrina, Paraná, coletaram essas duas espécies com atividade noturna e pico crepuscular. Esse mesmo tipo de resultado com relação a $C o$. venezuelensis, tinha sido observado por FORATTINI et al. (1981) e LOURENÇO-DEOliveira \& Silva (1985). Na Guiana Francesa, a espécie mostrou atividade durante o dia todo, mas predominando à noite, com pico logo após o pôr do sol (DÉGALLIER et al. 1978).

Mansonia titillans é conhecida como uma espécie crepuscular e noturna (HORSFALl 1955; ForATTINI 1965). BURTON (1964), na Guiana Francesa, encontrou-a com hábito diurno. DÉGALLIERI et al. (1978), observaram populações elevadas durante o dia e à noite, com pico não pronunciado após o por do sol. LOPES et al. (1995a) encontraram essa espécie em superpopulação na área periurbana de Londrina e observaram que a atividade hematofágica teve início ao escurecer, com pico logo após o por do sol. Porém, conforme o dia solar ia se tornando mais longo, o horário do pico ia se retardando e não mostrava mais correspondência com o crepúsculo. Mesmo após o pico, a população manteve-se alta.

A detecção de comportamento alimentar bimodal, que não foi observado em nenhuma espécie coletada nesta pesquisa, pode ter sido prejudicada pela queda da temperatura na segunda metade do período noturno das coletas. Essa diminuição da temperatura nas madrugadas é fator comum para o sul do Brasil, podendo assim, para esta região, não existir ou ser fraco o pico de picada ao amanhecer.

\section{CONCLUSÕES}

Anopheles strodei, An. galvaoi, An. evansae, An. albitarsis, Coquillettidia juxtamansonia, Co. venezuelensis, Mansonia titillans e Psorophora confinnis mostraram-se fortemente antropófilas e adaptadas ao ambiente antrópico.

Anopheles parvus, An. lutzii, An. oswaldoi, Culex (Melanoconion) sp., Cx. amazonensis e Aedes scapularis mostraram-se pouco adaptadas ao ambiente antrópico. Como as densidades medidas foram baixas, pouco pode-se afirmar sobre o grau de antropofilia dessas espécies.

A densidade populacional, muito acima da média anual, observada no mês de setembro em Anopheles (Nyssorhyncus) fortalece a hipótese de que as espécies deste subgênero passem o inverno na forma larvária.

Observou-se uma variação nas densidades populacionais durante o ano, quando An. strodei predominou de maio a dezembro. No verão e início do outono a predominância alternou-se entre $C x$.(Melanoconion) sp., Coquillettidiajuxtamansonia e Aedes fluviatilis.

Aedes fluviatilis, Anopheles argyritarsis, Culex coronator, Cx. mollis, Cx. quinquefasciatus e Psorophora cingulata embora tenham sidas coletadas em baixa freqüência neste experimento, são espécies conhecidas como domiciliadas ou em avançado processo de domiciliação. 
A atividade hematofágica desses Culicidae está concentrada no crepúsculo, não ocorrendo pico ao amanhecer. A ausência de maiores concentrações no amanhecer pode ser justificada pela diminuição da temperatura na segunda metade da noite.

A não captura de Aedomyia squamipennis com isca humana, embora as coletas tenham sido realizadas ao lado de um criadouro dessa espécie, comprova a ausência total de antropofilia da mesma.

AGRADECIMENTOS. Ao Dr. Oswaldo Paulo Forattini e Dra. Maria Anice Mureb Sallum da Faculdade de Saúde Pública, Universidade de São Paulo, pela confirmação da identificação das espécies de Culicidae.

\section{REFERÊNCIAS BIBLIOGRÁFICAS}

BARRETO, M.P. 1938. Observações sobre a ecologia de A. darlingi var. paulistensis. In: R.R. CORRÊA (Ed.). O Anopheles (N.) strodei Root, 1926 como provável vetor da malária. Rev. Biol. Hig. 9: 104-109.

BURTON, G.L. 1964. Attack on the vector of filariasis in British Guiana. Public Health Rep., Washington, 79: 137-143.

Consoli, R.A.G.B. 1940. Influência da salinidade no desenvolvimento de Aedes fluviatilis (Lutz, 1904) em laboratório (Diptera, Culicidae). Rev. Bras. Biol., Rio de Janeiro, 40 (1): 39-43.

Consoli, R.A.G.B.; P. Williams. 1981. A spects of the biology of laboratory-reared female Aedes fluviatilis. Mosq. News, Lake Charles, 41 (1): 30-36.

CORRÊA, R.R.O. 1938. Anopheles (N.) strodei Root, 1962 como provável vetôr de malária. Rev. Biol. Hyg. 9 (2): 104-109.

CoRrÊA, R.R.; A.S. Ramos. 1941. Do encontro de $A$. (N.) darlingi Root, 1926, e do $A$. (N.) oswaldoi var. metcalfi Galvão e Lane, 1938, naturalmente infectados com os parasitas maláricos, na região sul do Estado de São Paulo. Folia Clin. Biol., São Paulo, 6: 183-191.

Degallier, N.; F.X. Pajot; R. Kramer; J. Claustre; S. Bellony \& F. Lepont. 1978. Rythmes d'activite des Culicidés de la Guyane Française (Diptera: Culicidae). Cash. Orstom Sér. Ent. Méd. et Parasitol. 16: 73-84.

ForatTinI, O.P. 1965. Entomologia Médica. São Paulo, EDUSP, 1965. vol. 2, 506 .

ForatTINI, O.P. \& A. DE C. GoMeS. 1988. Biting activity patterns of Culex (Melanoconion) ribeirensis in southern Brazil. J. Am. Mosq. Control. Assoc., Lake Charles, 4 (2): 175-178.

ForATTINI, O.P.; O. DE S. LOPES \& E.X. RABELLO. 1968. Investigações sôbre o comportamento de formas adultas de mosquitos silvestres no Estado de São Paulo, Brasil. Rev. Saúde Pública, São Paulo, 2 (2): 11-173.

ForatTINI, O.P.; A. DE C. GoMES; D. NATAL \& J.L.F. SANTOS. 1986a. Observações sobre atividade de mosquitos Culicidae em mata primitiva da encosta no Vale 
do Ribeira, São Paulo, Brasil. Rev. Saúde Pública, São Paulo, 20 (1): 1-20.

1986b. Observações sobre atividade de mosquitos Culicidae em matas primitivas da planície e perfis epidemiológicos de vários ambientes no Vale do Ribeira, São Paulo, Brasil. Rev. Saúde Pública, São Paulo, 20 (3): 178-203.

Forattini, O.P.; G.K. Ishiata; E.X. Robello \& M. DAS D. Cotrim. 1973. Observação sobre os mosquitos Culex da cidade de São Paulo. Rev. Saúde pública, São Paulo, 7: 315-330.

ForatTINI, O.P.; A. DE C. GOMES; E.A.B. GALATI; E.X. RABELLO \& L.B. IVERSON. 1978a. Estudos ecológicos sobre mosquitos Culicidae no sistema da serra do mar, Brasil. I. Observações no ambiente extradomiciliar. Rev. Saúde Pública, São Paulo, 12: 297-325.

1978b. Estudos ecológicos sobre mosquitos Culicidae no sistema da serra do mar, Brasil. II. Observações no ambiente domiciliar. Rev. Saúde Pública, São Paulo, 12: 476-496.

Forattini, O.P.; A. DE C. Gomes; D. NATAL; I. KaKitAMI \& D. MARUCCI. 1987a. Preferências alimentares de mosquitos Culicidae no Vale do Ribeira, São Paulo, Brasil. Rev. Saúde Pública, São Paulo, 21 (3): 171-187.

1987b. Frequência domiciliar e endofilia de mosquitos Culicidae no Vale do Ribeira, São Paulo, Brasil. Rev. Saúde Pública, São Paulo, 21 (3): 188-192. 1989. Preferfências alimentares e domiciliação de mosquitos Culicidae no Vale do Ribeira, São Paulo, Brasil, com especial referência a Aedes scapularis e a Culex (Melanoconion). Rev. Saúde Pública, Sâo Paulo, 23 (1): 9-19.

Forattini, O.P.; A. DE C. GoMes; J.L.F. SANTOS; E.A.B. Galati; E.X. Rabello \& D. NATAL. 1981. Observações sobre atividade de mosquitos Culicidae, em mata residual no Vale do Ribeira, São Paulo, Brasil. Rev. Saúde Pública, São Paulo, 15: 557-586.

Gabaldon, A.; G. UlloA; N. Godoy; E. Marquez \& J. Pulido. 1977. Aedeomyia squamipennis (Diptera, Culicidae) vector natural de malaria aviaria en Venezuela. Bol. Dir. Malariol. y Saneamiento Ambiental, Caracas, 17 (1): 9-13.

GAlvão, A.A.; L. LANE \& R. CORRÊA. 1937. Notas sobre os Nyssorhynchus São Paulo. V. Sobre os Nyssorhynchus de Novo Oriente. Rev. Biol. Hyg., São Paulo, 8 (1): $37-45$.

Gomes, A. DE C. \& O.P. Forattini. 1990. Abrigos de mosquitos Culex (Culex) em zona rural (Diptera: Culicidae). Rev. Saúde Pública, São Paulo, 24 (5): 394-397.

Gomes, A. DE C.; O.P. ForatTini \& D. NATAL. 1987. Composição e atividade de mosquitos Culicidae. Emprego de Armadilha CDC no Vale do Ribeira, Estado de São Paulo, Brasil. Rev. Saúde Pública, São Paulo, 21 (5): 363-370.

Guimarães, A.E. \& V.M.N. Victorio. 1986. Mosquitos no Parque nacional da

Serra dos Órgãos do Estado do Rio de Janeiro, Brasil. III. Preferência horária para hematofagia. Mem. Inst. Oswaldo Cruz, Rio de Janeiro, 81 (1): 93-103. Guimarães, A.E.; M. Arlé \& R.N.M. Machado. 1985. Mosquitos no Parque 
Nacional da Serra dos Órgãos, Estado do Rio de Janeiro, Brasil. II. Distribuição vertical. Mem. Inst. Oswaldo Cruz, Rio de Janeiro, 80 (2): 171-185.

Horsfall, W.R. 1955. Mosquitoes: their bionomics and relation to disease. The Ronald Press Co., 384p.

CAlisher, C.H.; J.S. Lazuick \& D. Sudia. 1988. Brus Laguna Virus, a Gamboa bunyavirus from Aedomyia squamipennis collected in Honduras. Am. J. Trop. Med. Hyg., Lawrence, 39 (4): 406-408.

JORDAN, H.B. 1961. The effects of the quality of blood and temperature on the production and viability of eggs in Culex quinquefasciatus. Mos. News, Lake Chales, 21 (2): 133-135.

LOPES, J. \& A.L. Lozover. 1995. Ecologia de mosquitos (Diptera: Culicidae) em criadouros naturais e artificiais de área rural do Norte do Paraná, Brasil. I. Coletas ao longo do leito do ribeirão. Rev. Saúde Pública, São Paulo, 29 (3): 183-91.

LoPES, J.; V.D.R.B. DE OliveiRa \& F.J. DE A. OliveIRA. 1995a. Dominância de Mansonia titillans (Diptera: Culicidae) em uma área urbana no sul do Brasil. Semina, Londrina, 16 (2).

LOPES, J.; F.J. DE A. OLIVEIRA; V.D.R.B. DE OLIVEIRA \& M.A.P. TONON. 1995 b. Alteração na densidade populacional e diversidade específica de Culicidae (Diptera) na área urbana do Município de Londrina, Estado do Paraná, Sul do Brasil em conseqüência de modificações ambientais. Semina, Londrina, 16 (2).

LOURENÇO-DE-OlIVEIRA, R. 1984. Alguns aspectos da ecologia dos mosquitos (Diptera: Culicidae) de uma área de planície (Granjas Calábria), em Jacarepaguá, Rio de Janeiro. I. Freqüência comparativa das espécies em diferentes ambientes e métodos de coleta. Mem. Inst. Oswaldo Cruz., Rio de Janeiro, 79 (4): 479-490.

LOURENÇO-DE-OlIVEIRA, R. \& T.F. DA SILVA. 1985. Alguns aspectos da ecologia dos mosquitos (Diptera: Culicidae) de uma área de planície (Granjas Calábria), em Jacarepaguá, Rio de Janeiro. III. Preferência horária das fêmeas para o Hematofagismo. Mem. Inst. Oswaldo Cruz, Rio de Janeiro, 80 (2): 195-201.

NeVES, D.P. \& J.E. DA Silva. 1976. Os Culicidae do Museu de História Natural da Universidade Federal de Minas Gerais, Belo Horizonte.II. Variação estacional dos mosquitos da mata natural e da de eucalipto. Rev. Brasil. Biol., Rio de Janeiro, 36: 555-560.

RACHOU, R.G. 1958. Anofelinos do Brasil: comportamento das espécies vetoras de malária. Rev. Bras. Malariol. Doencas Trop., Brasília, 10: 145-181.

RACHOU, R.G.; M.M. LIMA; J.A.F. NETO \& C.M. MARTINS. 1958. Alguns dados sobre o comportamento de mosquitos de Ponta Grossa (Florianópolis, Santa Catarina). Rev. Brasil. Malariol. Doenças Trop., Brasília, 10: 417-427.

Recebido em 08.V.1995; aceito em 28.X.1996. 